\title{
Diabetic macular edema with pachychoroid features
}

\author{
Kyungeun Kang ${ }^{\dagger}$, Hyungwoo Lee ${ }^{\dagger}$, Minsu Jang, Hyung Chan Kim and Hyewon Chung ${ }^{*}$
}

\begin{abstract}
Background: To investigate the clinical features of diabetic macular edema (DME) in eyes with pachychoroid phenotypes using multimodal retinal imaging.

Methods: We retrospectively reviewed 210 eyes from 210 DME patients and analyzed the clinical and imaging parameters, including visual acuity, central macular thickness (CMT), subfoveal choroidal thickness (SFCT) and neural retina layer thickness (NRT). The DME eyes were divided into two groups: group 1 (80 eyes with submacular detachment [SMD]) and group 2 (130 eyes without SMD). The clinical and imaging parameters of 285 eyes from 285 diabetic patients without DME were collected as a control group.

Results: DME eyes with pachychoroid phenotypes were more frequent in group 1 than in group 2 (53 eyes [66.25\%] and 53 eyes [40.77\%], respectively, $P<0.001)$. Pachychoroid phenotypes were identified in 108 (37.90\%) of the control eyes. CMT and NRT were greater in group 1 than in group 2. In group 1, 37 eyes had SMD combined with focal edema, and 43 eyes had SMD combined with diffuse-type edema. No significant difference in pachychoroid phenotypes was found between the focal and diffuse types (26 [70.27\%] and 27 [62.79\%], respectively, $P=0.481$ ). In group 2, 70 eyes had focal-type edema, and 60 eyes had diffuse-type edema. No significant difference in the frequency of pachychoroid phenotypes was found (32 [45.71\%] and 21 [35.00\%], respectively, $P=0.215)$. Interestingly, among the 70 eyes with focal edema in group 2, 13 (40.6\%) and 5 (13.2\%) eyes with and without pachychoroid phenotypes showed no definite microaneurysms, respectively.
\end{abstract}

Conclusion: SMD and focal edema without definite microaneurysms may be clinical manifestations of DME with pachychoroid phenotypes and possibly related to choroidal circulation disturbance in DME.

Keywords: Diabetic retinopathy, Diabetic macular edema, Pachychoroid, Central macula thickness, Subfoveal choroidal thickness

\section{Background}

Diabetic macular edema (DME) is the most common cause of vision loss in diabetic retinopathy (DR), and its prevalence increases up to $28-29 \%$ in patients with a diabetes duration greater than 20 years [1]. Since Otani et al. [2] reported three patterns of DME, namely, diffuse swelling, cystoid macular edema (CME) and serous macular detachment (SMD), using optical coherence

\footnotetext{
* Correspondence: hchung@kuh.ac.kr

${ }^{\dagger}$ Kyungeun Kang and Hyungwoo Lee contributed equally to this work. Department of Ophthalmology, Konkuk University School of Medicine, Konkuk University Medical Center, 120-1 Neungdong-ro, Gwangjin-gu, Seoul 05030, Republic of Korea
}

tomography (OCT), the clinical manifestations of each type of DME, including detailed morphologic classifications, have been described [3]. OCT can discriminate DME types, including those with SMD, which is difficult to diagnose by biomicroscopy or fluorescein angiography (FA) [4]. More recently, diffuse and focal edema have been described based on the different pathophysiologies of DME development: diffuse edema is thought to result from disrupted retinal vessel integrity induced by the generalized breakdown of the inner blood-retinal barrier (BRB) [5], whereas focal edema results from microaneurysms, with pathology observed primarily in the outer retina [6]. When diffuse leakage occurs in the inner retina,

(c) The Author(s). 2020 Open Access This article is licensed under a Creative Commons Attribution 4.0 International License, which permits use, sharing, adaptation, distribution and reproduction in any medium or format, as long as you give appropriate credit to the original author(s) and the source, provide a link to the Creative Commons licence, and indicate if changes were made. The images or other third party material in this article are included in the article's Creative Commons licence, unless indicated otherwise in a credit line to the material. If material is not included in the article's Creative Commons licence and your intended use is not permitted by statutory regulation or exceeds the permitted use, you will need to obtain permission directly from the copyright holder. To view a copy of this licence, visit http://creativecommons.org/licenses/by/4.0/ The Creative Commons Public Domain Dedication waiver (http://creativecommons.org/publicdomain/zero/1.0/) applies to the data made available in this article, unless otherwise stated in a credit line to the data. 
fluid collects mainly in the inner nuclear layer (INL), resulting in a diffuse edema pattern.

Among the various patterns of DME, the importance of SMD in diffuse or focal edema has not been adequately depicted. Although the prevalence of SMD is quite high, approximately ranging from 10 to $30 \%$ [2, 4, $7,8]$, the pathogenesis and functional consequences of SMD remain controversial $[4,8,9]$. Inner and/or outer BRB compromise are the main causes for the development of macular edema in diabetic eyes; however, the genesis of SMD in these eyes seems involve a complex process. The inner plexiform layer (IPL) and outer plexiform layer (OPL) constitute 2 diffusion barriers of intraretinal fluid distribution [6]. The excessive intraretinal fluid from the disruption of the inner BRB has been hypothesized to reach the subretinal space because the external limiting membrane (ELM) is not impermeable to fluid and albumin $[3,10,11]$. If a large water load is not removed properly by the retinal pigment epithelium (RPE) (failure of the RPE pump), SMD could occur. One good example of SMD occurrence from inner BRB breakdown is in cases of retinal vein occlusion (RVO); the prevalence of SMD in RVO is much higher than that in DME. Excess fluid that reaches the subretinal space from rapidly increased intraretinal fluid in RVO might exceed the RPE active transport mechanism [2, 7]. However, this mechanism of SMD development from inner BRB disruption hardly explains the occurrence of SMD without substantial retinal fluid accumulation or when DME is focal (fluid in only the outer plexiform/outer nuclear layer [OPL/ONL]) and far from the macula [8], especially the nasal location. Thus, subretinal fluid accumulation may occur in DME with outer BRB breakdown, and choroidal circulation also leaks into the subretinal space.

There have been different reports regarding choroidal thickness (CT) during DR and DME. Some authors reported that the choroid thins during the progression of DR, reflecting decreased choroidal circulation and vascular insufficiency in DR and DME [12-16] whereas others reported no significant association between CT and DR or DME [17, 18]. In contrast, some authors found that CT increased with the severity of DR and in eyes with DME $[19,20]$. There could be several explanations for these divergent reports, including not adjusting for age and refractive error and not using enhanced depth imaging OCT (EDI OCT), but studies of heterogeneous eyes with different prevailing pathophysiologies involved in the development of DME might be a major reason. Choroidal thickening is a manifestation observed in various disorders affecting the retina and choroid, including recently described pachychoroid spectrum diseases. Pachychoroid spectrum diseases, including pachychoroid pigment epitheliopathy (PPE), pachychoroid neovasculopathy (PNV), and central serous chorioretinopathy (CSC), have common characteristics: diffuse or focal areas of increased CT, dilated choroidal vessels (pachyvessels), and thinning or absence of inner choroid (the choriocapillaris [CC] and Sattler's layer) overlying pachyvessels [21-25]. Although a number of studies have described various manifestations of these diseases, their pathophysiologies remain debatable. In particular, the influence of pachychoroid features on the manifestations of various forms of DME has not been studied.

Thus, this study was designed to determine the clinical manifestations of DME with or without pachychoroid features using multimodal retinal imaging. In this study, we investigated whether particular types of macular edema are more common in diabetic eyes with pachychoroid phenotypes.

\section{Methods}

\section{Study population}

A total of 2272 consecutive eyes with DR from 1136 diabetic patients who were followed up at Konkuk University Medical Center, Seoul, Korea, from November 2016 to October 2018 were included. For this retrospective study, we reviewed the medical records for 2272 eyes of 1136 patients. Only one eye was selected in each patient; if both eyes had DME, the first eye with this condition or the worse eye at presentation was selected. If patients had no macular edema in both eyes, the eye that had less media opacity and clearer images was selected. In 2272 eyes, 978 eyes were excluded because EDI OCT was not performed at the initial examination. A total of 444 eyes were excluded for the following reasons: vitreous hemorrhage, epiretinal membrane, vitreomacular traction and high myopia more than 6 diopters. A total of 298 eyes with a history of other eye diseases that may affect the outcome, such as RVO and age-related macular degeneration above the intermediate stage, were also excluded. In addition, we excluded 57 patients who had a history of uveitis, glaucoma, other previous intraocular surgery except for cataract and refractive surgery and past treatments such as laser photocoagulation or intravitreal injection. Finally, we included 285 eyes with no DME and 210 eyes with DME from among 495 eyes with DR.

A total of 495 eyes were divided into five groups by two retinal specialists. When the specialists disagreed while assigning the eyes to groups, they reviewed the patient charts together and reached a consensus. Eyes without DME were assigned to the control group. Group 1 was defined as DME with SMD. Group 1 was further divided into groups $1 \mathrm{~A}$ and $1 \mathrm{~B}$ according to the presence of focal or diffuse edema, respectively. As previously described [2, 6], focal edema was defined as retinal fluid collection only in the outer retina (the OPL to the ELM). In diffuse edema, retinal fluid is located in the inner retina (the internal 
limiting membrane [ILM] to the INL). Combined-type edema, where fluid is observed in the inner and outer retina, was also designated as diffuse edema. Thus, diffuse edema was defined as retinal fluid located in the inner retina with or without retinal fluid in the outer retina. Group 2 consisted of DME eyes without SMD. Group 2A was defined as focal-type edema, and group $2 \mathrm{~B}$ was defined as diffuse-type edema. Finally, each group was further divided into two subgroups according to the presence or absence of pachychoroid features.

All patients underwent a comprehensive ophthalmological examination at the first visit, including measurement of the best-corrected visual acuity (BCVA) using a Snellen chart, intraocular pressure, slit-lamp biomicroscopy, fundus examination, and color fundus photography using Topcon TRC-50IX (Topcon Medical Systems Inc., Paramus, New Jersey, USA) or Optos P200Tx (Optos plc., Dunfermline, Scotland, UK). EDI OCT images were obtained using a Spectralis HRA + $\mathrm{OCT}^{\bullet}$ device (Spectralis; Heidelberg Engineering, Heidelberg, Germany). Some patients underwent other tests, including FA, ICGA, and OCT angiography (OCTA).

Pachychoroid was defined as a subfoveal choroidal thickness (SFCT) of $300 \mu \mathrm{m}$ or more and at least one of the following characteristics based on previous studies [21-25]: the existence of enlarged choroidal vessels (pachyvessels) and/or attenuated inner choroid on EDI OCT or choroidal hyperpermeability on ICGA.

\section{Image analysis}

Volume scans generated by SD OCT images at baseline were selected for analyses. These scans covered a $9 \mathrm{~mm} \times 6 \mathrm{~mm}$ area and contained 25 scans spaced $238 \mu \mathrm{m}$ apart. Our first goal of image analysis was to calculate the SFCT and inner and outer retina thicknesses. The choroid thickness was measured from the outer portion of the hyperreflective line corresponding to the RPE to the inner surface of the sclera on the EDI OCT image. Using the caliper tool available from Heidelberg, SFCT was measured perpendicularly $3000 \mu \mathrm{m}$ wide in the subfoveal choroid centered on the fovea. The central macular thickness (CMT), inner retina layer (IPL/INL) and outer retina layer (OPL/ONL) were manually measured using manual segmentation. CMT was defined within a $1000 \mu \mathrm{m}$ diameter, and inner and outer retina layer thicknesses were measured within the $3000 \mu \mathrm{m}$ diameter cylinder at the macula. En face images of the $\mathrm{CC}$ were obtained by using OCTA with autosegmentation.

All of these processes were managed primarily by a trained ophthalmologist (K Kang) and further confirmed by a retinal specialist ( $\mathrm{H}$ Chung).

\section{Statistical analyses}

Statistical analyses were performed using IBM SPSS software ver. 18.0 for windows (IBM Corp, Chicago, IL, USA). Visual acuity obtained using a Snellen chart was switched to the logarithm of the minimal angle of resolution (logMAR) BCVA for statistical analyses. Pearson's Chi-square test was performed to compare the ratio of sex and prevalence of pachychoroid, hypertension and dyslipidemia. Fisher's exact test was performed to compare the prevalence of cardiovascular disease and kidney disease history. One-way ANOVA was performed to evaluate the difference in age. The Kruskal-Wallis test with the Mann-Whitney $U$ test was used to compare the CMT, SFCT, BCVA (logMAR), IPL/INL thickness and OPL/ONL thickness. $P<0.05$ was considered statistically significant.

\section{Results}

This study included 495 eyes of 495 patients with DR (mean age $58.66 \pm 12.69$ years, 304 men and 191 women). The number of eyes with pachychoroid features in each group is described in Table 1 . In the control group and groups 1A, 1B, 2A and 2B, 108 (37.9\%), 26 (70.3\%), 27 (62.8\%), 32 (45.7\%) and 21 (35.0\%) eyes had pachychoroid phenotypes, respectively. The frequency of pachychoroid phenotypes was significantly higher in group 1 than in the control group $(P<0.001)$. The ratio of pachychoroid phenotypes was significantly higher in group $1(66.3 \%)$ than in group $2(40.8 \%, P<0.001)$. The demographic characteristics of the patients from each group are shown in Table 1. There were no significant differences in age, sex, and the prevalence of hypertension, dyslipidemia, cardiovascular diseases and kidney diseases among the groups.

The mean $\log$ MAR BCVA at baseline was $0.16 \pm 0.28$. Among all groups, the BCVA in the control group (0.07 \pm 0.17 ) was better than that in the other groups, and the $\mathrm{BCVA}$ in group $1 \mathrm{~B}(0.36 \pm 0.38)$ was worse than that in the other groups. In the DME groups, no significant difference in BCVA was found between groups 1A, 1B, 2A and 2B (ANOVA, $P=0.516$ ) (Fig. 1a). A similar trend was observed only when patients with pachychoroid phenotypes were analyzed (Fig. 1a); the BCVA in the control group $(0.08 \pm 0.23)$ was better than that in the other groups, and the BCVA in group $1 \mathrm{~B}(0.37 \pm 0.46)$ was worse than that in the other groups. In DME eyes, no significant difference in $\mathrm{BCVA}$ was found between groups $1 \mathrm{~A}, 1 \mathrm{~B}, 2 \mathrm{~A}$ and $2 \mathrm{~B}$ (Kruskal-Wallis test, $P=0.107$ ).

\section{Comparison of CMT, SFCT and inner and outer retina layer thicknesses among the groups with pachychoroid features}

CMT and SFCT in all groups are shown in Table 2. When only eyes with pachychoroid phenotypes from 
Table 1 Baseline characteristics of each group

\begin{tabular}{|c|c|c|c|c|c|c|c|}
\hline & $\begin{array}{l}\text { Control }(n= \\
285)\end{array}$ & $\begin{array}{l}\text { Group 1A } \\
(n=37)\end{array}$ & $\begin{array}{l}\text { Group 1B } \\
(n=43)\end{array}$ & $\begin{array}{l}\text { Group 2A } \\
(n=70)\end{array}$ & $\begin{array}{l}\text { Group 2B } \\
(n=60)\end{array}$ & $\begin{array}{l}p- \\
\text { value }\end{array}$ & $\begin{array}{l}\text { Total }(n= \\
\text { 495) }\end{array}$ \\
\hline $\begin{array}{l}\text { Non-pachychoroid/Pachychoroid } \\
\text { (number) }\end{array}$ & $177 / 108$ & $11 / 26$ & $16 / 27$ & $38 / 32$ & $39 / 21$ & $\begin{array}{l}< \\
0.001\end{array}$ & $281 / 214$ \\
\hline Sex (male/female) & $167 / 118$ & $26 / 11$ & $30 / 13$ & $48 / 22$ & $33 / 27$ & 0.424 & $304 / 191$ \\
\hline Age $($ mean $\pm S D)$ & $59.41 \pm 13.64$ & $57.08 \pm 11.35$ & $55.74 \pm 10.94$ & $57.87 \pm 11.30$ & $59.05 \pm 11.33$ & 0.385 & $\begin{array}{l}58.66 \pm \\
12.69\end{array}$ \\
\hline Prevalence of HTN (number, \%) & $144(50.5)$ & $17(45.9)$ & $21(48.8)$ & $36(51.4)$ & $29(48.3)$ & 0.420 & $247(49.9)$ \\
\hline $\begin{array}{l}\text { Prevalence of dyslipidemia (number, } \\
\%)\end{array}$ & $56(19.6)$ & $5(13.5)$ & $8(18.6)$ & $14(20.0)$ & $6(10.0)$ & 0.429 & $89(18.0)$ \\
\hline Prevalence of CVA Hx (number, \%) & $42(14.7)$ & $6(2.7)$ & $2(4.7)$ & $6(8.6)$ & $5(8.3)$ & 0.185 & $61(12.3)$ \\
\hline $\begin{array}{l}\text { Prevalence of kidney disease } \mathrm{Hx} \\
\text { (number, \%) }\end{array}$ & $19(6.6)$ & $4(10.8)$ & $6(14.0)$ & $8(11.4)$ & $4(6.7)$ & 0.376 & $41(8.3)$ \\
\hline
\end{tabular}

Control, no diabetic macular edema (DME)

Group 1, serous macular detachment (SMD), further subdivided into group 1A with focal edema and group 1B with diffuse edema

Group 2A, focal edema type without SMD

Group 2B, diffuse edema type without SMD

SD standard deviation, HTN hypertension, CVA Hx cardiovascular disease history (including cerebral infarction, a history of cardiac arrest, angina, previous CABG, and previous $\mathrm{PCl}$ )

One-way ANOVA (age), Pearson's Chi-square test (pachychoroid type, sex, hypertension, dyslipidemia) and Fisher's exact test (CVA Hx, kidney disease Hx) were used

DME patients were analyzed (groups 1A, 1B, 2A and 2B), CMT was greatest in group $1 \mathrm{~B}(431.00 \mu \mathrm{m}$, $500.48 \mu \mathrm{m}, 345.38 \mu \mathrm{m}$ and $402.95 \mu \mathrm{m}$ in groups $1 \mathrm{~A}, 1 \mathrm{~B}$, $2 \mathrm{~A}$ and $2 \mathrm{~B}$, respectively, $P<0.001)$. A significant difference in CMT was found between all groups except between groups $1 \mathrm{~A}$ and $2 \mathrm{~B}$ and between groups $2 \mathrm{~A}$ and $2 \mathrm{~B}$ $(P=0.831,0.164$, respectively) (Table 2$)$. No significant difference in SFCT was found among groups 1A, 1B, $2 \mathrm{~A}$ and $2 \mathrm{~B}(P=0.297)$ (Table 2$)$.

Among the eyes with pachychoroid phenotypes, IPL/ INL and OPL/ONL thicknesses were greatest in group 1B $(91.52 \mu \mathrm{m}, 111.41 \mu \mathrm{m}, 82.36 \mu \mathrm{m}$ and $98.31 \mu \mathrm{m}$ in groups $1 \mathrm{~A}, 1 \mathrm{~B}, 2 \mathrm{~A}$ and $2 \mathrm{~B}$, respectively, $P<0.001$ for IPL/INL thickness; $175.78 \mu \mathrm{m}, 199.83 \mu \mathrm{m}, 135.09 \mu \mathrm{m}$

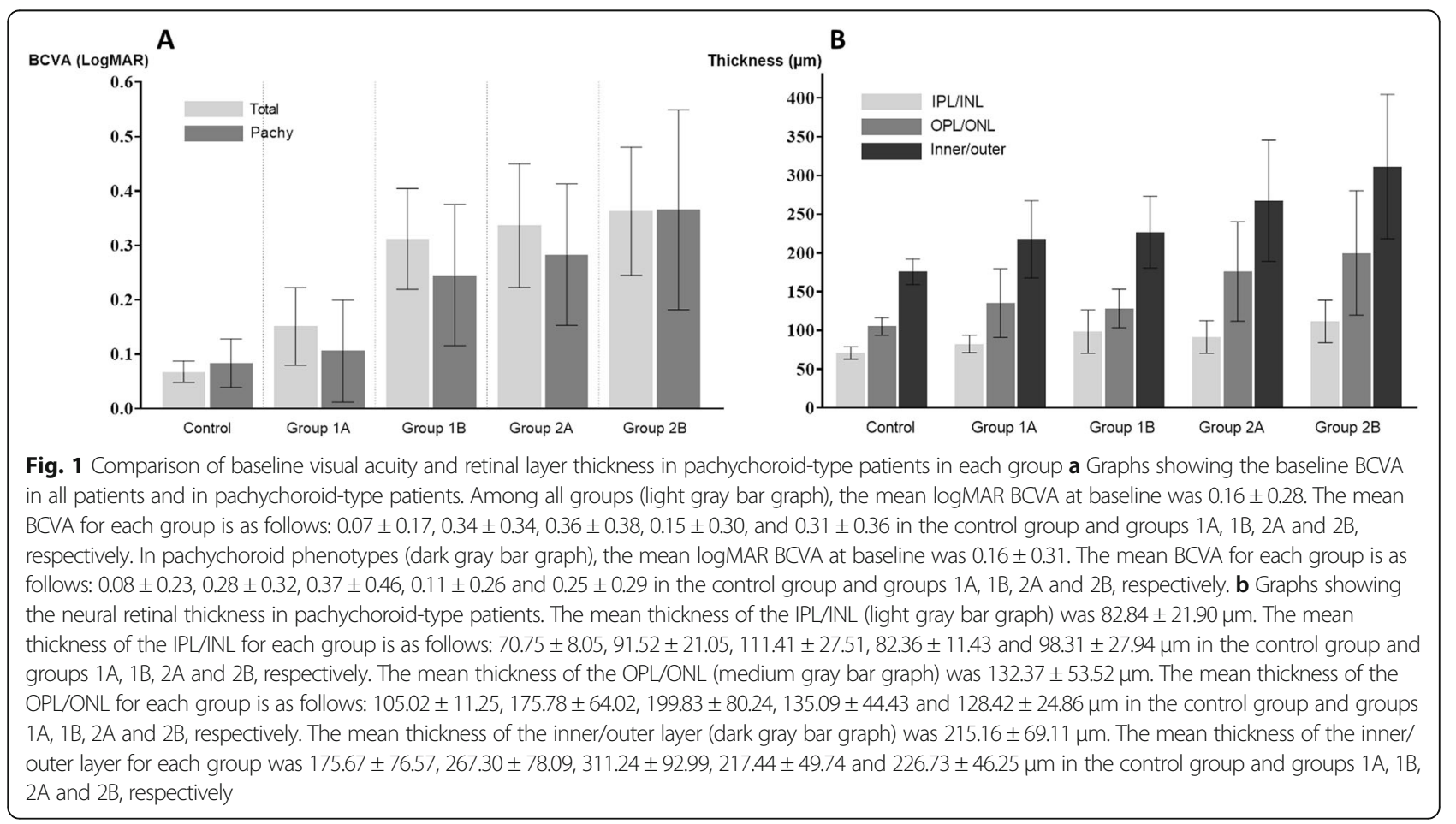


Table 2 Comparison of central macular thickness (CMT) and subfoveal choroidal thickness (SFCT)

\begin{tabular}{|c|c|c|c|c|c|c|c|}
\hline & $\begin{array}{l}\text { Control } \\
(n=285)\end{array}$ & $\begin{array}{l}\text { Group 1A } \\
(n=37)\end{array}$ & $\begin{array}{l}\text { Group 1B } \\
(n=43)\end{array}$ & $\begin{array}{l}\text { Group 2A } \\
(n=70)\end{array}$ & $\begin{array}{l}\text { Group 2B } \\
(n=60)\end{array}$ & $p$-value & $\begin{array}{l}\text { Total } \\
(n=495)\end{array}$ \\
\hline \multicolumn{8}{|l|}{ All eyes } \\
\hline $\mathrm{CMT}(\mu \mathrm{m} \pm \mathrm{SD})$ & $267.29 \pm 24.44$ & $445.89 \pm 133.11^{*}$ & $532.47 \pm 161.65^{*} \dagger$ & $336.21 \pm 82.93^{*} † \neq$ & $408.22 \pm 106.32^{*} \neq \S$ & $<0.001$ & $330.50 \pm 117.30$ \\
\hline $\mathrm{SFCT}(\mu \mathrm{m} \pm \mathrm{SD})$ & $273.41 \pm 95.82$ & $320.89 \pm 71.97^{*}$ & $310.70 \pm 82.04$ & $296.14 \pm 87.52$ & $279.25 \pm 100.16$ & 0.006 & $284.12 \pm 93.54$ \\
\hline \multicolumn{8}{|c|}{ Eyes with pachychoroid } \\
\hline CMT $(\mu \mathrm{m} \pm \mathrm{SD})$ & $265.92 \pm 24.57$ & $431.00 \pm 139.97^{*}$ & $500.48 \pm 169.68^{*} \dagger$ & $345.38 \pm 80.82^{*} \dagger \neq$ & $402.95 \pm 105.92^{*} \neq \S$ & $<0.001$ & $340.90 \pm 124.80$ \\
\hline $\mathrm{SFCT}(\mu \mathrm{m} \pm \mathrm{SD})$ & $374.33 \pm 56.20$ & $360.08 \pm 35.11$ & $357.59 \pm 56.74$ & $370.56 \pm 55.67$ & $388.14 \pm 70.68$ & 0.297 & $371.28 \pm 55.82$ \\
\hline
\end{tabular}

*Statistically significant $(p<0.05)$ compared to control group

†Statistically significant $(p<0.05)$ compared to group $1 \mathrm{~A}$

\#Statistically significant $(p<0.05)$ compared to group 1B

$\S$ Statistically significant $(p<0.05)$ between group $2 \mathrm{~A}$ vs $2 \mathrm{~B}$

$S D$ standard deviation

One-way ANOVA and post hoc tests (CMT, SFCT) were used

Bold values denote statistical significance $(p<0.05)$

and $128.42 \mu \mathrm{m}$ in groups $1 \mathrm{~A}, 1 \mathrm{~B}, 2 \mathrm{~A}$ and $2 \mathrm{~B}$, respectively, Kruskal-Wallis test, $P<0.001$ for OPL/ONL thickness) (Fig. 1b). In group 1, no eyes with focal DME (26 eyes) or diffuse DME (27 eyes) showed normal neuroretinal thickness (within the $3000-\mu \mathrm{m}$ central subfield) above the SMD. The highest point of thickening within the $3000-\mu \mathrm{m}$ central subfield in the neural retina was significantly higher in eyes with SMD (groups $1 \mathrm{~A}$ and $1 B$ ) than that in eyes without SMD (groups $2 \mathrm{~A}$ and $2 \mathrm{~B}$ ) (Fig. 1b).

Intriguingly, in group 2A, definite microaneurysms resulting in OPL/ONL edema were not identified in 13 eyes among 32 eyes with pachychoroid phenotypes (40.6\%) by OCT and FA (Table 3 ). This percentage was higher than that of eyes with no identified microaneurysms and without pachychoroid phenotypes in group 2A (5 among 38 eyes [13.2\%] without pachychoroid phenotypes in group $2 \mathrm{~A}$ had no definite microaneurysms) (Pearson's chi-square test, $P=0.009$ ). Among 38 eyes without pachychoroid features, 33 eyes with microaneurysms showed higher CMT than did 5 eyes without definite microaneurysms (342.79 and $251.20 \mu \mathrm{m}, P=0.010$ ), confirming that leakage from microaneurysms contributed to macular edema in these patients. Among 18 eyes without definite microaneurysms, 13 eyes with pachychoroid features showed higher CMT than did 5 eyes without pachychoroid features (358.23 and $251.20 \mu \mathrm{m}$, $P=0.019$ ). When we analyzed 52 eyes with microaneurysms or 52 eyes in which we considered that a leak from microaneurysms was the major mechanism of their macular edema (focal-type edema), the CMT of 19 eyes with pachychoroid features was not different from that of 33 eyes without pachychoroid features (336.58 and $342.79 \mu \mathrm{m}, P=0.676)$. However, BCVA was better in 19 eyes with pachychoroid features than in 33 eyes without pachychoroid features $(0.02$ and $0.20, P=0.044)$. Finally, among 32 eyes with pachychoroid features, no differences were found in CMT or other OCT parameters

Table 3 Comparison of the presence of microaneurysm (Ma) in group 2A

\begin{tabular}{|c|c|c|c|c|c|c|c|c|}
\hline & \multicolumn{4}{|c|}{ Group 2A $(n=70)$} & \multirow[b]{3}{*}{$\begin{array}{l}p \text { - } \\
\text { value* }\end{array}$} & \multirow[b]{3}{*}{$\begin{array}{l}p \text { - } \\
\text { valuet }\end{array}$} & \multirow[b]{3}{*}{$\begin{array}{l}p- \\
\text { valuef }\end{array}$} & \multirow[b]{3}{*}{$\begin{array}{l}\text { Total } \\
(n=70)\end{array}$} \\
\hline & \multicolumn{2}{|c|}{$\begin{array}{l}\text { Non-pachychoroid type } \\
(n=38)\end{array}$} & \multicolumn{2}{|c|}{$\begin{array}{l}\text { Pachychoroid type } \\
(n=32)\end{array}$} & & & & \\
\hline & $\begin{array}{l}\mathrm{Ma}(-) \\
(n=5)\end{array}$ & $\begin{array}{l}\mathrm{Ma}(+) \\
(n=33)\end{array}$ & $\begin{array}{l}\mathrm{Ma}(-) \\
(n=13)\end{array}$ & $\begin{array}{l}\text { Ma (+) } \\
(n=19)\end{array}$ & & & & \\
\hline CMT $(\mu \mathrm{m} \pm \mathrm{SD})$ & $251.20 \pm 67.58$ & $342.79 \pm 81.28$ & $358.23 \pm 115.08$ & $336.58 \pm 47.11$ & 0.061 & 0.010 & 0.019 & $337.43 \pm 166.61$ \\
\hline $\mathrm{SFCT}(\mu \mathrm{m} \pm \mathrm{SD})$ & $219.80 \pm 69.90$ & $236.24 \pm 52.27$ & $384.46 \pm 64.86$ & $361.05 \pm 47.92$ & $<0.001$ & 0.645 & $<0.001$ & $296.47 \pm 87.46$ \\
\hline $\begin{array}{l}\text { Thickness of INL/IPL }(\mu \mathrm{m} \pm \\
\text { SD) }\end{array}$ & $401.40 \pm 37.45$ & $424.88 \pm 151.43$ & $431.77 \pm 73.20$ & $398.11 \pm 39.54$ & 0.831 & 0.967 & 0.703 & $417.21 \pm 110.65$ \\
\hline $\begin{array}{l}\text { Thickness of ONL/OPL }(\mu \mathrm{m} \pm \\
\text { SD) }\end{array}$ & $609.80 \pm 86.02$ & $665.91 \pm 160.43$ & $715.92 \pm 291.83$ & $647.74 \pm 161.88$ & 0.862 & 0.769 & 0.443 & $666.26 \pm 186.55$ \\
\hline BCVA (logMAR) $($ mean $\pm S D)$ & $0.15 \pm 0.31$ & $0.20 \pm 0.34$ & $0.23 \pm 0.22$ & $0.02 \pm 0.26$ & 0.099 & 0.331 & 0.387 & $0.15 \pm 0.30$ \\
\hline
\end{tabular}

Ma microaneurysm, CMT central macular thickness, SFCT subfoveal choroidal thickness, INL inner nuclear layer, IPL inner plexiform layer, ONL outer nuclear layer, OPL outer plexiform layer, BCVA best-corrected visual acuity

Symbols depict the $p$-values between the total group $\left({ }^{*}\right)$, the non-pachychoroid type without Ma, the pachychoroid type with Ma( $\dagger$ ) and the pachychoroid types with and without $\mathrm{Ma}(\ddagger)$

The Kruskall-Wallis test with the Mann-Whitney $U$ test was applied

Bold values denote statistical significance $(p<0.05)$ 
measured between 13 eyes without definite microaneurysms and 19 eyes with microaneurysms (Table 3 ).

\section{Findings on ICGA and OCTA in diabetic eyes with pachychoroid features}

Among 106 eyes with pachychoroid phenotypes (106 DME eyes with pachychoroid phenotypes in groups $1 \mathrm{~A}$, $1 \mathrm{~B}, 2 \mathrm{~A}$ and $2 \mathrm{~B}$ ) and ICGA results, 47 eyes showed early hypofluorescent lesions in the early phase and choroidal hyperpermeability in the late phase. Good-quality OCTA images of the CC slab were obtained for 49 eyes among the 106 eyes. In groups $1 \mathrm{~A}, 1 \mathrm{~B}, 2 \mathrm{~A}$ and $2 \mathrm{~B}, 68.75,40.00$, 42.86 and $66.67 \%$ of eyes showed areas devoid of flow at the CC slab on OCTA, respectively (Supplemental Fig. 1, $2,3,4)$.

\section{Discussion}

In the present study, pachychoroid phenotypes were more frequent in diabetic eyes with SMD than in those without SMD. We also observed a certain type of focal edema that might be derived from direct RPE leakage and is associated with pachychoroid phenotypes (Supplemental Fig. 1 and 3). Progressive thickening of the choroid or a thickened choroid with the progression of DR or the presence of DME has been reported $[8,13,20]$. SMD is reportedly related to increased CT, increased CC permeability and outer BRB dysfunction. Based on the findings of the present study, we suggest that there may be specific types of DME associated with pachychoroid characteristics in addition to CT changes along with DR and/or DME. This idea can also explain the very divergent reports regarding $\mathrm{CT}$ and $\mathrm{DR}$ and/or DME thus far [12-20]. Proteinaceous exudate in the subretinal space might be at least partially caused by excessive leakage from the CC.

When we analyzed eyes with pachychoroid phenotypes in the control and groups $1 \mathrm{~A}, 1 \mathrm{~B}, 2 \mathrm{~A}$ and $2 \mathrm{~B}$, no significant difference in SFCT or BCVA was found between group $1 \mathrm{~B}$ and group 2B. However, CMT and the thickness of the inner/outer retinal layers were significantly higher in group $1 \mathrm{~B}$ (all $P<0.001$ ). Additionally, no difference in SFCT or BCVA was found between group 1A and group 2A. Nevertheless, CMT and the thickness of the inner/outer retinal layers were significantly larger in group 1A than in group 1B (all $P<0.001$ ). Thus, SMD may develop with large neural retinal fluid accumulation, but no correlation was found between the presence of SMD and visual acuity, consistent with findings in other studies $[8,11,26]$. As Gaucher et al. [8] described, functional impairment of the RPE and choroidal changes could lead to SMD in diabetic eyes, in addition to insufficient passive resorption of intraretinal fluid for the development of SMD. Nonperfusion of the CC causes ischemic damage or necrosis of the RPE and breakdown of outer BRB, resulting in the development of SMD [12, 13]. Loss of the CC or decreased choroidal blood flow may result in a hypoxic environment around the RPE, followed by upregulation of VEGF production by the RPE [8, 27]. In histologic studies of the diabetic choroid, choroidal neovascularization $(\mathrm{CNV})$ that was associated with areas of CC loss at or peripheral to the equator was observed [28]. FA and ICGA of these patients show filling delay of the choroid, indicating $\mathrm{CC}$ nonperfusion in the early phase and multiple hyperfluorescent spots in the middle to late phase [29]. In our study, macular ischemia, which appears as decreased CC flow on OCTA, was detected in $14(42.86 \%)$ and $9(66.67 \%)$ eyes in groups $1 \mathrm{~A}$ and $1 \mathrm{~B}$, respectively. As Gaudric et al. [30] hypothesized, if choroidal ischemia is moderate, passage of fluid through the RPE could lead to SMD; in contrast, RPE cell death occurs with a greater extent of choroidal ischemia.

Interestingly, among 18 eyes without definite microaneurysms in group 2A (the focal edema group), we noted that 13 eyes with pachychoroid features showed greater CMT than that in 5 eyes without pachychoroid features ( 358.23 and $251.20 \mu \mathrm{m}, P=0.019$, Table 3 ), suggesting that an RPE leak accompanied by pachychoroid phenotypes may be a major contributing mechanism for macular edema in these patients. No difference in BCVA between these two subgroups was found $(P=0.387$, Table 3$)$. When we analyzed 52 eyes with microaneurysms in which a leak from microaneurysms was the major mechanism of macular edema, there was no difference in CMT between 19 eyes with pachychoroid features and 33 eyes without pachychoroid features (336.58 and $342.79 \mu \mathrm{m}, P=0.676)$. However, BCVA was better in 19 eyes with pachychoroid features than in 33 eyes without pachychoroid features (0.02 and $0.20, P=0.044)$. These findings collectively suggest that eyes with edema from an RPE leak in pachychoroid phenotypes could develop during the course of DR. Thus, these features might not necessarily indicate a chronic course or a poorer prognosis. Remarkably, 7 of 18 eyes without definite microaneurysms in group $2 \mathrm{~A}$ showed intraretinal fluid accumulation in the nasal macula (Supplemental Fig. 3). Regarding the possible mechanism associated with this unusual location of macular edema in eyes with DME, these eyes may share overlapping features with peripapillary pachychoroid syndrome (PPS), a recently described type of pachychoroid spectrum disease [31]. Although 4 of 16 cases with PPS in the study by Phasukkijwatana et al. [31] had underlying DM, it remains unclear whether DM and/or DME with pachychoroid features can be included in the clinical spectrum of PPS, which is characterized by peripapillary choroidal thickening associated with nasal macular intraretinal and/or subretinal fluid and occasional disc edema. Although the 
pathophysiological mechanisms of PPS are not completely known [29], similar or shared mechanisms of RPE dysfunction and subsequent fluid leakage into the intraretinal space due to high hydrostatic pressure from peripapillary choroidal congestion are likely operating in our nasally located focal DME eyes with pachychoroid features and without microaneurysms.

This study has several limitations. First, the study design is retrospective, and only images and data collected at the initial presentation were analyzed. Future longitudinal studies including the association of changes in OCT parameters after the treatment of DME are needed. However, the strengths of the present study included a large number of consecutive DR patients in one tertiary hospital. In addition, DME patients were divided into four groups, and the analysis was further conducted on subgroups according to the presence or absence of pachychoroid phenotypes for the first time. Second, the definition of pachychoroid phenotypes has not been clarified; however, we have defined them as defined in previous studies as much as possible [21-25]. Finally, OCTA examination was not conducted on all patients; thus, the definite relationship between $\mathrm{CC}$ ischemia and outer retinal damage or edema should be evaluated in future studies. In addition, OCTA could possibly be used to find MA that were not observed in OCT or FA. However, a recent study reported that FA counted a significantly higher number of MAs compared with the five different OCTA devices, including ours (Spectralis OCTA, Heidelberg, Germany) [32]. When compared with FA, Spectralis OCTA showed a sensitivity of $43 \%$, specificity of $55 \%$, positive predictive value (PPV) of $81 \%$ and negative predictive value (NPV) of $17 \%$. Due to the lower PPV and NPV, OCTA still has weaknesses in defining the presence or absence of MAs. Another study reported that MAs that appear hyporeflective on structural OCT have a lower detection rate on OCTA [33]. Nevertheless, the possibility of finding new MAs with OCTA exists, and large population studies will be needed in the future.

\section{Conclusions}

In conclusion, SMD and focal edema from RPE leakage may be clinical manifestations of DME with pachychoroid phenotypes. A better understanding of choroidal disturbances in DME is important for accurate evaluation and treatment of DME.

\section{Supplementary information}

Supplementary information accompanies this paper at https://doi.org/10. 1186/s12886-020-01663-y.

Additional file 1: Figure S1. A representative case of the focal-edema type with serous macular detachment (group 1A).
Additional file 2: Figure S2. A representative case of the diffuseedema type with serous macular detachment (group 1B).

Additional file 3: Figure S3. A representative case of the focal-edema type without serous macular detachment (group 2A).

Additional file 4: Figure S4. A representative case of the diffuseedema type without serous macular detachment (group 2B).

\section{Abbreviations}

DME: Diabetic macular edema; DR: Diabetic retinopathy; CME: Cystoid macular edema; SMD: Submacular detachment; OCT: Optical coherence tomography; FA: Fluorescein angiography; BRB: Blood-retinal barrier; INL: Inner nuclear layer; IPL: Inner plexiform layer; OPL: Outer plexiform layer; CMT: Central macular thickness; ELM: External limiting membrane; RPE: Retinal pigment epithelium; RVO: Retinal vein occlusion; CT: Choroidal thickness; SFCT: Subfoveal choroidal thickness; NRT: Neural retina layer thickness; EDI OCT: Enhanced depth imaging OCT; PPE: Pachychoroid pigment epitheliopathy; PNV: Pachychoroid neovasculopathy; CSC: Central serous chorioretinopathy; CC: Choriocapillaris; ILM: Internal limiting membrane; PPS: Peripapillary pachychoroid syndrome; PPV: Positive predictive value; NPV: Negative predictive value

\section{Acknowledgements}

Not Applicable.

\section{Authors' contributions}

$\mathrm{HC}$ contributed to the design of the study. $\mathrm{HL}, \mathrm{HCK}$ and $\mathrm{HC}$ provided the patient care. KK and $\mathrm{MJ}$ collected the patient data regarding the outcomes presented in this paper. $\mathrm{KK}, \mathrm{HL}$ and $\mathrm{HC}$ contributed to the analysis and interpretation of the data. $\mathrm{KK}, \mathrm{HL}$ and $\mathrm{HC}$ contributed to the writing of the manuscript. $\mathrm{HC}$ contributed to the acquisition of funding and general supervision. KK, MJ, HCK and HC contributed to the statistical analyses. KK, $\mathrm{HL}, \mathrm{MJ}, \mathrm{HCK}$ and $\mathrm{HC}$ contributed the revision of the manuscript. All authors have read and approved the final manuscript.

\section{Funding}

This study was supported by the National Research Foundation of Korea and funded by the Ministry of Science and ICT [NRF-2019M3A9H1030948]. The funding body was not involved in the design of the study and collection, analysis, and interpretation of data or in the writing of the manuscript.

\section{Availability of data and materials}

All data generated or analyzed during the current study are available from the corresponding author on reasonable request.

\section{Ethics approval and consent to participate}

The study was conducted in accordance with the tenets of Declaration of Helsinki. This study was approved as exempt by the local Institutional Review Board/Ethics Committee at Konkuk University Medical Center due to the retrospective nature of the study and the lack of identifiable data. Access of the data used in this research was permitted by the Institutional Review Board/Ethics Committee at Konkuk University Medical Center (KUH1 100058).

Consent for publication

Not Applicable.

Competing interests

The authors declare that they have no competing interests.

Received: 14 May 2020 Accepted: 27 September 2020

Published online: 02 October 2020

References

1. Klein R, Klein BE, Moss SE, Davis MD, DeMets DL. The Wisconsin epidemiologic study of diabetic retinopathy. IV Diabetic macular edema. Ophthalmology. 1984;91:1464-74.

2. Otani T, Kishi S, Maruyama Y. Patterns of diabetic macular edema with optical coherence tomography. Am J Ophthalmol. 1999;127:688-93.

3. Kang SW, Park CY, Ham DI. The correlation between fluorescein angiographic and optical coherence tomographic features in clinically significant diabetic macular edema. Am J Ophthalmol. 2004;137:313-22. 
4. Ozdemir H, Karacorlu M, Karacorlu S. Serous macular detachment in diabetic cystoid macular oedema. Acta Ophthalmol Scand. 2005;83:63-6.

5. Bresnick GH. Diabetic maculopathy. A critical review highlighting diffuse macular edema. Ophthalmology. 1983;90:1301-17.

6. Byeon SH, Chu YK, Hong YT, Kim M, Kang HM, Kwon OW. New insights into the pathoanatomy of diabetic macular edema: angiographic patterns and optical coherence tomography. Retina. 2012;32:1087-99.

7. Koleva-Georgieva D, Sivkova N. Assessment of serous macular detachment in eyes with diabetic macular edema by use of spectral-domain optical coherence tomography. Graefes Arch Clin Exp Ophthalmol. 2009;247:1461-9.

8. Gaucher D, Sebah C, Erginay A, Haouchine B, Tadayoni R, Gaudric A, et al. Optical coherence tomography features during the evolution of serous retinal detachment in patients with diabetic macular edema. Am J Ophthalmol. 2008;145:289-96.

9. Gungel $\mathrm{H}$, Altan $\mathrm{C}$. The relationship between fundus fluorescein angiographic findings and serous macular detachment in diabetic macular edema. Retina. 2013:33:1895-901.

10. Ossewaarde-van Norel J, Berg EM, Sijssens KM, Rothova A. Subfoveal serous retinal detachment in patients with uveitic macular edema. Arch Ophthalmol. 2011;129:158-62.

11. Shereef H, Comyn O, Sivaprasad S, Hykin P, Cheung G, Narendran N, et al. Differences in the topographic profiles of retinal thickening in eyes with and without serous macular detachment associated with diabetic macular oedema. Br J Ophthalmol. 2014;98:182-7.

12. Adhi M, Alwassia AA, Duker JS. Analysis of choroidal thickness in eyes treated with focal laser photocoagulation using SD-OCT. Can J Ophthalmol. 2013:48:535-8.

13. Campos A, Campos EJ, Martins J, Ambrosio AF, Silva R. Viewing the choroid: where we stand, challenges and contradictions in diabetic retinopathy and diabetic macular oedema. Acta Ophthalmol. 2017:95:446-59.

14. Melancia D, Vicente A, Cunha JP, Abegao Pinto L, Ferreira J. Diabetic choroidopathy: a review of the current literature. Graefes Arch Clin Exp Ophthalmol. 2016;254:1453-61.

15. Unsal E, Eltutar K, Zirtiloglu S, Dincer N, Ozdogan Erkul S, Gungel H. Choroidal thickness in patients with diabetic retinopathy. Clin Ophthalmol. 2014;8:637-42

16. Horvath H, Kovacs I, Sandor GL, Czako C, Mallar K, Recsan Z, et al. Choroidal thickness changes in non-treated eyes of patients with diabetes: sweptsource optical coherence tomography study. Acta Diabetol. 2018;55:927-34.

17. Esmaeelpour M, Povazay B, Hermann B, Hofer B, Kajic V, Hale SL, et al. Mapping choroidal and retinal thickness variation in type 2 diabetes using three-dimensional 1060-nm optical coherence tomography. Invest Ophthalmol Vis Sci. 2011;52:5311-6.

18. McCourt EA, Cadena BC, Barnett CJ, Ciardella AP, Mandava N, Kahook MY. Measurement of subfoveal choroidal thickness using spectral domain optical coherence tomography. Ophthalmic Surg Lasers Imaging. 2010; 41(Suppl):S28-33.

19. Hua R, Liu L, Wang X, Chen L. Imaging evidence of diabetic choroidopathy in vivo: angiographic pathoanatomy and choroidal-enhanced depth imaging. PLoS One. 2013;8:e83494.

20. Kim JT, Lee DH, Joe SG, Kim JG, Yoon YH. Changes in choroidal thickness in relation to the severity of retinopathy and macular edema in type 2 diabetic patients. Invest Ophthalmol Vis Sci. 2013:54:3378-84.

21. Chung $H$, Byeon SH, Freund KB. Focal choroidal excavation and its association with pachychoroid spectrum disorders: a review of the literature and multimodal imaging findings. Retina. 2017;37:199-221.

22. Dansingani KK, Balaratnasingam C, Naysan J, Freund KB. En face imaging of pachychoroid spectrum disorders with swept-source optical coherence tomography. Retina. 2016:36:499-516.

23. Gallego-Pinazo R, Dolz-Marco R, Gomez-Ulla F, Mrejen S, Freund KB. Pachychoroid diseases of the macula. Med Hypothesis Discov Innov Ophthalmol. 2014;3:111-5.

24. Pang CE, Freund KB. Pachychoroid neovasculopathy. Retina. 2015;35(1):1-9.

25. Warrow DJ, Hoang QV, Freund KB. Pachychoroid pigment epitheliopathy. Retina. 2013;33:1659-72.

26. Bolz M, Ritter M, Schneider M, Simader C, Scholda C, Schmidt-Erfurth U. A systematic correlation of angiography and high-resolution optical coherence tomography in diabetic macular edema. Ophthalmology. 2009;116:66-72.

27. Nagaoka T, Kitaya N, Sugawara R, Yokota H, Mori F, Hikichi T, et al. Alteration of choroidal circulation in the foveal region in patients with type 2 diabetes. Br J Ophthalmol. 2004;88:1060-3.
28. McLeod DS, Lutty GA. High-resolution histologic analysis of the human choroidal vasculature. Invest Ophthalmol Vis Sci. 1994;35:3799-811.

29. Aoyagi R, Hayashi T, Tsuneoka H. Choroidal thickening and macular serous retinal detachment in pregnancy-induced hypertension. Int Med Case Rep J. 2015;8:291-4

30. Gaudric A, Coscas G, Bird AC. Choroidal ischemia. Am J Ophthalmol. 1982; 94:489-98.

31. Phasukkijwatana N, Freund KB, Dolz-Marco R, Al-Sheikh M, Keane PA, Egan CA, et al. Peripapillary pachychoroid syndrome. Retina. 2018;38:1652-67.

32. Parrulli S, Corvi F, Cozzi M, et al. Microaneurysms visualisation using five different optical coherence tomography angiography devices compared to fluorescein angiography. Br J Ophthalmol. 2020. https://doi.org/10.1136/ bjophthalmol-2020-316817.

33. Parravano M, De Geronimo D, Scarinci F, Querques L, Virgili G, Simonett J, et al. Diabetic microaneurysms internal reflectivity on spectral-domain optical coherence tomography and optical coherence tomography angiography detection. Am J Ophthalmol. 2017;179:90-6.

\section{Publisher's Note}

Springer Nature remains neutral with regard to jurisdictional claims in published maps and institutional affiliations.
Ready to submit your research? Choose BMC and benefit from:

- fast, convenient online submission

- thorough peer review by experienced researchers in your field

- rapid publication on acceptance

- support for research data, including large and complex data types

- gold Open Access which fosters wider collaboration and increased citations

- maximum visibility for your research: over $100 \mathrm{M}$ website views per year

At $\mathrm{BMC}$, research is always in progress.

Learn more biomedcentral.com/submissions 